\title{
NEW EQUATION TO ESTIMATE GLOMERULAR FILTRATION RATE IN CHINA: A REFERENCE ISSUE
}

\author{
Pierre Delanaye ${ }^{1}$ and Hans Pottel ${ }^{2}$ \\ ${ }^{1}$ Department of Nephrology-Dialysis-Transplantation, University of Liège (ULg CHU), CHU Sart Tilman, Liège, \\ Belgium; and ${ }^{2}$ Department of Public Health and Primary Care, KU Leuven Campus Kulak Kortrijk, Kortrijk, Belgium
}

To the editor: From a large cohort $(\mathrm{n}=8571)$, Li et al. ${ }^{1}$ proposed a new creatinine-based equation to estimate glomerular filtration rate (GFR) in Chinese adults, using ${ }^{99 \mathrm{~m} T \mathrm{Tc}-\mathrm{DTPA}}$ (technetium diethylenetriamine pentaacetic acid) renal dynamic imaging as the reference method. This new equation performed better than others. However, validation is performed with the same reference method in a similar population by the same researchers, and we therefore question the use of the term "external validation." It can indeed be expected that the new equation would perform better when used in a population similar to the one used for its development. In the 2 so-called "external" validation samples, the percentage of estimated GFR predictions within 30\% of measured GFR (P30) was 75.19\% and $77.02 \%$, respectively, and this is still far below P30s observed in other large studies. The P30s of the Chronic Kidney Disease-Epidemiology Collaboration and full age spectrum equations were close to $85 \%{ }^{2,3} \mathrm{Also}$, this relatively poor performance can be explained by the way GFR has been measured. In our opinion, $99 \mathrm{mTc}$-DTPA plasma or urinary clearance might be considered as references, although dynamic imaging is able to give only a split function (\% of marker uptake by left and right kidney), but not a GFR result. None of the references cited by Li et al. ${ }^{1}$ actually supports dynamic imaging for GFR measurement. ${ }^{4}$ On the contrary, Xie et al. ${ }^{5}$ showed, in 149 Chinese subjects with GFR measured by ${ }^{99 \mathrm{~m} T c-D T P A}$ plasma clearance, that the performance of $99 \mathrm{mTc}$-DTPA dynamic imaging produced a less accurate GFR than the GFR estimated according to the Chronic Kidney Disease-Epidemiology Collaboration equation (P30 of $66.44 \%$ and $71.14 \%$, respectively). Significant improvement in the performance of eGFR equations can be expected only from studies with an adequate GFR measurement.

1. Li D-y, Yin W-j, Yi Y-h, et al. Development and validation of a more accurate estimating equation for glomerular filtration rate in a Chinese population. Kidney Int. 2019;95:636-646.

2. Levey AS, Stevens LA, Schmid CH, et al. A new equation to estimate glomerular filtration rate. Ann Intern Med. 2009;150:604-612.

3. Pottel H, Hoste L, Dubourg L, et al. A new estimating glomerular filtration rate equation for the full age spectrum. Nephrol Dial Transplant. 2016;31: 798-806.

4. Blaufox MD, Aurell M, Bubeck B, et al. Report of the Radionuclides in Nephrourology Committee on renal clearance. J Nucl Med.1996;37:1883-1890.

5. Xie P, Huang JM, Liu XM, et al. (99m)Tc-DTPA renal dynamic imaging method may be unsuitable to be used as the reference method in investigating the validity of CDK-EPI equation for determining glomerular filtration rate. PLoSOne. 2013;8:e62328. 